\title{
Cardiac surgery for communities in need - meeting the continuous challenges for delivering new models of global humanitarian health programmes
}

Abbreviations: WHO, world health organization; CEIF, cardiac eye international foundation

\section{Editorial}

According to the World Health Organization (WHO), out of a thousand surviving neonates at least eight present congenital heart diseases. The National Health Organization states annually that between 6000-7000 patients undergo surgical intervention in order to correct acquired and congenital cardiac defects each year. Yet only a tiny fraction of patients without health insurance are fortunate enough to be operated in hospitals associated to health insurance companies each year in third world countries. ${ }^{1,2}$ This is mainly because "Medical Tourism" has emerged as a new trend where people from developed countries travel to places like India, Brazil, Thailand, and Malaysia for a range of medical procedures including cardiac bypass surgery. Private hospitals with Western-trained physicians have mushroomed in cities across these countries to specifically provide services to foreigners.

Surgeries in these hospitals cost a fraction of what they would for similar services in the West. However, for many poor patients living in many developing countries in Asia, Latin America and Africa this model of venture capital-driven health care is beyond reach. Nowadays, several actions aimed at implementing humanitarian programs to support non-affording patients in health systems around the world have been proposed, especially in the pediatric setup. In the recent gathering of pediatric cardiac surgeons at the World Summit on Pediatric and Congenital Heart Surgery, held in Geneva in June $2015,{ }^{3,4}$ it was possible to mobilize Cardiac Surgery Groups and multidisciplinary groups of health experts from wealthy countries planning to perform humanitarian missions in Third World Countries.

Numerous governmental and non-governmental agencies have provided excellent humanitarian medical assistance for a large number of populations in need. Nevertheless, to date, this assistance has been "reactive" and tokenistic. It has mainly been offered in response to natural disasters and wars. These programs have certainly been useful, but have only been of a punctual, time-limited nature and therefore not offered significant long-term capacity-building solutions. ${ }^{3-5}$

With the primary aim of participating in philanthropic activities that focused on addressing health needs for children on long waiting lists for surgical intervention we put together a multi-disciplinary team through an umbrella organization called Cardiac Eye International Foundation (CEIF). Initially we sought to provide free diagnostic and surgical operational assistance to non-affording patients with congenital and acquired cardiovascular disease. Very soon it became clear that CEIF would achieve tangible solutions if we could focus our efforts on improving access to quality pediatric and adult cardiac care by helping to develop from the ground cardiac programs in areas of need as sustainable centers of excellence. We then worked in collaboration with many other dedicated medical volunteers from around the world (Australia, Chile, United Kingdom, Kenya United
Volume 3 Issue I - 2016

\author{
Maqsood M Elahi,' Bashir M Matata',2,3 \\ 'Heart-Lung \& Research Institute, Cardiac Eye International \\ Foundation, USA \\ ${ }^{2}$ Cardiac Eye International Foundation Africa, Kenya \\ ${ }^{3}$ Liverpool Heart \& Chest Hospital, United Kingdom
}

Correspondence: Bashir Matata, Liverpool Heart \& Chest Hospital,Thomas Drive, Liverpool, LI 4 3PE,

United Kingdom,Email matata_bashir@hotmail.com

Received: February 02, 2016 | Published: February 05, 2016

States and Pakistan), as well as other organizations to provide free complex heart surgeries, training, mentoring, capacity building, opportunities that are designed to meet the inimitable needs of our partner sites.

Our work with partner sites focuses mainly on building of clinical and organizational capability through training and mentorship. Our phased support approach envisions promoting growth and improving quality of care to international cardiac surgery standards, particularly in children and adults. There is therefore a gradual shift away from the practice of sponsoring indigent children and young adults to undergo open heart surgery in selected fee-paying centers overseas. Instead we focus on and harnessing local talent, sponsoring open heart surgery teams to facilitate the development, support, or augment with similar teams in the host countries as our primary goal. All patients get clinical evaluation performed by an adult or pediatric cardiologist within the team. This also includes post-operative consultation with the chief surgeon for any additional examinations if required and preparedness for the post-operative and follow-up care of such patients.

Since the inauguration debut in 2009 one of the main missions for CEIF was to explore ways for delivering accessible and affordable cardiac surgery for poor patients in Pakistan under the CEIF Pakistan So far 57 patients (both adults and children) have undergone free open heart surgeries at two medical facilities in Lahore, Pakistan. From then on, future missions were planned. On-site surgical missions (both solo and team efforts) were initiated, augmented by efforts to modernize cardiac surgery programmes through health visits, lectures, consultations and cooperative efforts all over the country. CEIF has also committed its resources to developing free centers of excellence for cardiac services in East Africa with a base in Mombasa, Kenya. CEIF with its donations from other partners is providing financial support to the charity program within Kenya and also for Nigerian patients requiring cardiac surgery. The plan is to implement free heart surgery services for patients with congenital heart defects in communities in need around the world in the next few years. 
The efforts have resulted in many adult and pediatric open heart operations being performed over the last 3 years as well as several closed and open cardiac and thoracic surgeries. In addition to the financial donations, we have also contributed training of healthcare professionals with an interest in participating in charitable cardiovascular disease treatment causes. We believe that it is only through this kind of multiplication of philanthropic and humanitarian actions together will we provide long term solutions to this serious health problem.

So far, the CEIF global initiative has been transformed into an international force for alleviating suffering in children and adults living with heart disease in relatively poor countries. In our experience, there are many obstacles besides the financial costs. To deliver these services we had to overcome many complex issues such as: political intransigence, corruptive practices, archaic beliefs and work place cultural hegemony. In particular, many government funded organizations we come across were chronically under-funded and lack the vision or leadership to explore other ways of delivering new services for communities in dire need. In contrast, private hospitals tend to pay less attention to non-affording patients and focus on medical tourism.

The strategy of focusing the CEIF network on developing countries has allowed us to maximize the collective power to make an impact in a sustainable way. Not only are we able to mobilize our network of free heart surgery programs to help centers in different countries in their efforts to reduce the number of children waiting for surgery but we are able to galvanize global awareness to create the context within a country for the development of a sustainable program.

We aim to continue with this programme of training missions and assisting hospitals to provide complex cardiac surgeries for free for the foreseeable future. We work with our partner sites and volunteers to identify opportunities to support and organize capacity building activities like;

i. Team training visits to address specialist skills development needs

ii. Local team work training and mentoring;

iii. Leadership and administrative systems development including distance learning. In addition to clinical skills, sustainable progress demands other types of skills and abilities such as leadership vision and capability, organizing finances, organizational culture, and support systems such as fundraising.
We believe that time has come to reflect on the past failures and focus on new achievements. We should challenge ourselves to create strong partnerships with local talent to build successful collaborations for the future. For this reason, international specialists in the area of humanitarian and cardiovascular medicine should be invited, as well as, representatives from international non-governmental organizations working in the area in order to further share their ideas and experience. By coming together, we are sure to find the best solutions for better health and quality of life to the poorer populations of the world.

\section{Acknowledgements}

None.

\section{Conflict of interest}

The author declares no conflict of interest.

\section{References}

1. World summit on pediatric and congenital heart surgery. Services, education and cardiac care in children and adults with congenital heart disease. Global Coalition to Improve Cardiac Care for Children and Adults with Pediatric and Congenital Heart Disease Across the World. Montreal, Canada; 2008.

2. Jenkins KJ. Risk adjustment for congenital heart surgery: the RACHS-1 method. Semin Thorac Cardiovasc Surg Pediatr Card Surg Annu. 2004;7:180-184.

3. John L. Surgeries, Side Trips for "Medical Tourists". In: Affordable Care at India's Private Hospitals Draws Growing Number of Foreigners. USA: Washington Post Foreign Service; 2004. A01 p.

4. Leung R. 60Minutes Story: Vacation, adventure and surgery, elective surgeries by world-class doctors at third-world prices. CBS Broadcast; 2005.

5. Novick WM, Stidham GL, Karl TR, et al. Are we improving after 10 years of humanitarian paediatric cardiac assistance? Cardiol Young. 2005;15(4):379-384. 